\title{
ASYMPTOTICS OF INTEGRODIFFERENTIAL MODELS WITH INTEGRABLE KERNELS
}

\author{
ANGELINA M. BIJURA
}

Received 12 September 2002

\begin{abstract}
The additive decomposition singular perturbation method and the theory of fractional integration are used to study asymptotic solutions of singularly perturbed Volterra integrodifferential equations with kernels having integrable singularity. The validity of the approximation is also demonstrated.
\end{abstract}

2000 Mathematics Subject Classification: 41A60, 45J05, 45E10, 26 A33.

1. Introduction. There are various kinds of singularities in mathematical problems. Problems which are considered here are those which depend on a parameter in such a way that regions of rapid variation occur, and where there is a drastic change in the structure of the problem when the limiting operation is performed. Assumptions are imposed on the data to exclude other behaviours. In particular, asymptotic solutions of singularly perturbed linear scalar Volterra integral equations with weakly singular kernels are investigated. Equations of the following form are considered:

$$
\varepsilon y^{\prime}(t)=g(t)+\frac{1}{\Gamma(1-\beta)} \int_{0}^{t} \frac{k(t, s)}{(t-s)^{\beta}} y(s) d s, \quad 0<t \leq T, y(0)=y_{0}(\varepsilon),
$$

where $0<\varepsilon \ll 1$ and $0<\beta<1$. The functions $g(t)$ and $k(t, s)$ are continuous and $k(t, t)<0,0 \leq t \leq T$. The initial condition $y_{0}(\varepsilon)$ is regular with respect to $\varepsilon$ as $\varepsilon$ tends to zero when $g(0)=0$ and singular when $g(0) \neq 0$. For this reason, it is appropriate to denote $y_{0}(\varepsilon)=y_{0}$ when $g(0)=0$ and $y_{0}(\varepsilon)=\tilde{y}_{0} / \xi(\varepsilon)$ when $g(0) \neq 0$, where $y_{0}$ and $\tilde{y}_{0}$ are constants and $\xi(\varepsilon) \rightarrow 0$ when $\varepsilon \rightarrow 0$. It will be demonstrated in Section 4 that the assumption $k(t, t)<0$ on $0 \leq t \leq T$ allows the solution $y(t ; \varepsilon)$ of (1.1) to have an initial layer and forces the algebraic decay in the layer region.

This problem exhibits an initial layer at $t=0$ like Volterra integral and integrodifferential equations with continuous kernels as well as integral equations with weakly singular kernels. However, in this case, it will be shown that there is a thicker initial layer width, of order $O\left(\varepsilon^{1 /(2-\beta)}\right)$, as $\varepsilon \rightarrow 0$. The kernel and the forcing function may as well depend on $\varepsilon$ but it is assumed here that they are independent of $\varepsilon$. The motivation to study this problem follows its 
rigorous application to numerous problems of mathematical physics, including problems described in Section 2.

The weakly singular equation (1.1) has a solution $y(t ; \varepsilon)$ in $C[0, T]$ for all $\varepsilon>0$. When $\varepsilon=0$, (1.1) reduces to the Abel integral equation

$$
0=g(t)+\frac{1}{\Gamma(1-\beta)} \int_{0}^{t} \frac{k(t, s)}{(t-s)^{\beta}} v(s) d s, \quad 0 \leq t \leq T
$$

which certainly does not have a continuous solution if $g(0) \neq 0$. The forcing function $g(t)$ must be smoother than the desired solution. Even if (1.2) has a solution $v(t)$ in $C^{0}[0, T]$, it may not approximate $y(t ; \varepsilon)$ uniformly for all $0 \leq t \leq T$ as $\varepsilon \rightarrow 0$, especially if $v(0) \neq \lim _{\varepsilon \rightarrow 0} y(0 ; \varepsilon)$. Therefore, the need is to develop methodologies for the construction of a uniformly valid asymptotic solution of problem (1.1).

The introduction of appropriate new independent variable(s) is a basic technique used in obtaining asymptotic solutions of singular perturbation problems. In the case considered here, a new variable is introduced in such a way that the integral operator is regularized. Then, in place of the solution $y(t ; \varepsilon)$ of (1.1), an asymptotic solution is thought in an additive form of the solution depending on the original independent variable which is valid away from the initial layer (hence called the outer solution) and the solution depending on a new variable, valid in the initial layer (the inner layer solution). This transforms the whole problem into simpler equations in terms of the inner and outer equations.

In contrast to singularly perturbed integral equations with continuous kernels, equations with weakly singular kernels have not received much attention. This is due to the fact that there are difficulties encountered in the singular perturbation analysis of equations with weakly singular kernels. Singularly perturbed integral and integrodifferential equations with continuous kernels have inner layer solutions which decay exponentially. This simplifies the analysis. Inner layer solutions corresponding to integral equations with weakly singular kernels decay algebraically (rather than exponentially) and therefore the analysis poses some complications including that of facing nonconvergent integrals. Thus, the rigorous theory of singularly perturbed Volterra integral and integrodifferential equations with weakly singular kernels is still far from complete. This paper presents a general method for analyzing asymptotic solutions of (1.1).

Asymptotic solutions of (1.1) have previously been considered in [1]. A variety of interesting examples have been solved in [1], however the results were not rigorously established. Moreover, the case $g(0) \neq 0$ is not covered in the analysis presented in [1]. Recently, [2] studied, rigorously, asymptotic solutions of singularly perturbed linear Volterra integral equations with weakly singular kernels using the additive decomposition method. It is shown in [2] that the inner layer solution can explicitly be written in terms of the Mittag-Leffler 
function which tends to zero algebraically at infinity. Adopting the techniques used in [2] here, the formal asymptotic solution for (1.1) is derived by revealing the structure of its contents and proof of asymptotic correctness presented. Hence, this shows the essential difference between the results in this paper and the results in [1].

The theory of fractional integration and the Laplace transform method will be employed in most parts of the presentation. There are abnormalities arising when balancing terms of similar orders of $\varepsilon$. This occurs especially when one tries to derive the first- (and higher-) order terms in the formal solution. It appears that higher-order terms depend on a particular value of $\alpha$. Hence, the paper restricts attention to the leading-order solution.

The paper is organized as follows. In Section 2, examples of models arising from singularly perturbed Volterra integrodifferential equations are discussed. In Section 3, the review of some of the results which are applied later in the presentation is outlined. These include notations, definitions, hypotheses imposed on the data, and asymptotic evaluation of integrals via integral transforms and fractional integration. In Section 4, the application of the additive decomposition technique to integral equations of the type (1.1) is described and the leading-order formal solution, $y_{0}(t ; \varepsilon)$, is derived under stated conditions. The contents of the derived formal solution are shown to elucidate some of their properties assumed in the derivation. This is done in Section 5. It is also shown in Section 5 that if the formal solution satisfies (1.1) approximately with a residual $\rho(t ; \varepsilon)$, then $\rho(t ; \varepsilon)=O(\varepsilon)$ as $\varepsilon \rightarrow 0$, uniformly for $0 \leq t \leq T$. The main result in this paper is presented in Section 6 , where it is proved that if $y_{0}(t ; \varepsilon)$ is a formal approximate solution of $(1.1)$, and $y(t ; \varepsilon)$ is the exact solution, then $\left|y(t ; \varepsilon)-y_{0}(t ; \varepsilon)\right|$ is uniformly of order $O\left(\varepsilon^{\alpha}\right)$ as $\varepsilon \rightarrow 0$, on $0 \leq t \leq T$. This supports the finding that (1.1) has a wider initial layer. Finally in Section 7 , the methodology is demonstrated by solving the heat problem which portrays the diffusion of a gas through a liquid. This model, which is described in Section 2, has the exact solution, it is only chosen for demonstration. The conclusion summarizing the results is presented after Section 7.

2. Motivation. The study on solutions of (1.1) is motivated by the study on various models of mathematical physics including the following.

(A) The remarkably useful equation; the heat equation. Particularly the boundary value problem

$$
\begin{gathered}
C_{x x}-C_{t}=0, \quad x>0, t>0, \quad C(x, 0)=0, \quad x \geq 0, \\
\varepsilon C_{x}(0, t)=\int_{0}^{t} C(0, s) d s-f(t), \quad 0<\varepsilon \ll 1, \\
\lim _{x \rightarrow \infty} C(x, t)=0, \quad t \geq 0 .
\end{gathered}
$$

This equation models the diffusion of a gas through a liquid with a surface dissipation effect which depends upon the cumulative level of concentration. 
The function $C(x, t)$ denotes the concentration. The smallness of $\varepsilon$ implies a low diffusivity of the system. The function $f(t)$ is the given input at the surface. The Laplace transform method can be used to write the solution $C(x, t)$ of (2.1) as

$$
\begin{aligned}
\varepsilon C(x, t)=\int_{0}^{t} & \frac{1}{\sqrt{\pi(t-s)}} \exp \left[\frac{-x^{2}}{4(t-s)}\right] \\
& \times\left\{f(s)-\int_{0}^{s} C(0, \sigma) d \sigma\right\} d s, \quad t>0, x \geq 0 .
\end{aligned}
$$

Observe from (2.2) that $C(x, t)$ can be obtained once $C(0, t)$ is known. Thus, it is appropriate to formulate a suitable problem for the investigation of $C(0, t)$. Taking $x=0$ above, one obtains a Volterra integrodifferential equation

$$
\varepsilon y^{\prime}(t)=\int_{0}^{t} \frac{1}{\sqrt{\pi(t-s)}}\{f(s)-y(s)\} d s, \quad t \geq 0,
$$

where

$$
y(t)=\int_{0}^{t} C(0, s) d s
$$

(B) An integrodifferential equation that models the motion of a linear viscoelastic material. These materials are characterized by constitutive relations which are functionals of the past history of the material. In particular, consider a one-dimensional model problem

$$
\begin{gathered}
u_{t t}(x, t)=\sigma_{x}(x, t)+f(x, t), \quad 0<x<1, t>0, \\
u(x, t)=\phi(x, t), \quad 0 \leq x \leq 1,-\infty<t \leq 0, \\
u_{t}\left(x, 0_{+}\right)=\psi(x), \quad 0 \leq x \leq 1, \\
u(0, t)=u(1, t)=0, \quad t \geq 0,
\end{gathered}
$$

where $u(x, t)$ is the displacement at time $t$ of the particle with reference position $x$ on $0 \leq x \leq 1, u_{x}$ is the strain, $\sigma$ is the stress, and $f$ is a prescribed body force. The given functions $f, \phi$, and $\psi$ are real valued. If the body is elastic, then the stress depends on the strain through a constitutive equation,

$$
\sigma(x, t)=\frac{1}{\varepsilon} \int_{\mathbb{R}^{+}} A(s) \frac{\partial}{\partial t} u_{x}(x, t-s) d s,
$$

where the smallness of $\varepsilon$ implies that a highly strained material is used. Using (2.6), (2.5) can be reduced to a scalar Volterra integrodifferential equation depending parametrically on $x$ and written in an abstract form as

$$
\varepsilon y^{\prime}(t)=\int_{0}^{t} A(t-s) L y(s) d s+g(t ; \varepsilon), \quad t>0, y(0)=y_{0},
$$


where

$$
L \chi=\chi^{\prime \prime}, \quad \chi \in\left\{\xi^{\prime \prime} \in L^{2}(0,2) ; \xi(0)=\xi(1)=0\right\}
$$

In (2.7), $A$ is the same function as in (2.6) and is the linear stress relaxation modulus assumed to be nonnegative, nonincreasing, convex on $(0, \infty)$, and integrable on $(0,1)$. The function $g$ includes functions $f, \psi$, and the strain history. For a detailed discussion on integrodifferential equations in viscoelasticity, see $[10,13]$.

Equations (2.3) and (2.7) are special cases of a general class of singularly perturbed Volterra integrodifferential equations with weakly singular kernels that are considered here.

\section{Mathematical preliminaries}

Definition 3.1. (i) The fractional integral of order $\gamma>0$ is defined by

$$
J^{\gamma} \phi(t):=\frac{1}{\Gamma(\gamma)} \int_{0}^{t}(t-s)^{\gamma-1} \phi(s) d s, \quad t>0, \gamma \in \mathbb{R}^{+} .
$$

(ii) Using the Laplace convolution between two functions, the above definition can also be represented as

$$
J^{\gamma} \phi(t):=\frac{t^{\gamma-1}}{\Gamma(\gamma)} * \phi(t), \quad t>0, \gamma \in \mathbb{R}^{+}
$$

(iii) If one uses the sign $\circ$ to denote a Laplace transform pair, then it follows from (3.2) that

$$
J^{\gamma} \phi(t) \circ \frac{\Phi(s)}{s^{\gamma}}, \quad \gamma>0, s \in \mathbb{C},
$$

where $\Phi(s)$ denotes the Laplace transform of $\phi(t)$.

(iv) The Mittag-Leffler function of order $\gamma$ is defined by

$$
E_{\gamma}\left(\lambda t^{\gamma}\right)=\sum_{n=0}^{\infty} \frac{\lambda^{n} t^{n \gamma}}{\Gamma(n \gamma+1)}, \quad t>0, \gamma>0, \lambda \in \mathbb{C} .
$$

3.1. Hypothesis. The following assumptions are to be used:

$\left(\mathrm{H}_{\beta}\right) \quad 0<\beta<1$;

$\left(\mathrm{H}_{k}\right) k(t, s)$ is a $C^{2}$ function on $0 \leq s \leq t \leq T$ with $k(t, t)=-1$;

$\left(\mathrm{H}_{g}\right)$ the function $g(t) \in C^{2}[0, T]$ such that $g^{\prime}(0)=0$. 
For the readers convenience, the results to be employed later in the presentation are stated below.

\subsection{Asymptotic evaluation of integrals by integral transforms} and fractional calculus

LEMMA 3.2. Consider the integrodifferential equation,

$$
\phi^{\prime}(t)=\kappa-\frac{t^{-\beta}}{\Gamma(1-\beta)} * \phi(t), \quad t>0, \phi(0)=\phi_{0} .
$$

Suppose $\kappa$ and $\beta$ are constants, $0<\beta<1$. Then $\phi(t)$ admits, to the leadingorder, the asymptotic relations

$$
\begin{gathered}
\phi(t) \sim \frac{\kappa t^{\beta-1}}{\Gamma(\beta)}+\frac{\phi_{0} t^{\beta-2}}{\Gamma(\beta-1)}, \quad t \rightarrow \infty, \\
\phi(t) \sim \phi_{0}+\kappa t, \quad t \rightarrow 0 .
\end{gathered}
$$

Proof. Let $\Phi(s), s \in \mathbb{C}$, denote the Laplace transform of $\phi(t)$. If one proceeds into solving (3.5) using the Laplace transform method,

$$
\Phi(s)=\frac{\phi_{0} s^{1-\beta}}{s^{2-\beta}+1}+\frac{\kappa s^{-\beta}}{s^{2-\beta}+1} .
$$

To obtain the inverse Laplace transform, the Laplace transform pair

$$
E_{\gamma}\left(-\lambda t^{\gamma}\right) \circ \frac{s^{\gamma-1}}{s^{\gamma}+\lambda}, \quad \gamma>0, \lambda \in \mathbb{C},
$$

developed in [4] is employed and one obtains

$$
\phi(t)=\phi_{0} E_{2-\beta}\left(-t^{2-\beta}\right)+\kappa \int_{0}^{t} E_{2-\beta}\left(-s^{2-\beta}\right) d s
$$

The asymptotic expansion of the Mittag-Leffler function at infinity, investigated in [2], implies that

$$
\begin{aligned}
\phi(t)= & \kappa \sum_{i=1}^{\infty}(-1)^{i+1} \frac{t^{1+(\beta-2) i}}{\Gamma(2-(2-\beta) i)} \\
& +\phi_{0} \sum_{i=1}^{\infty}(-1)^{i+1} \frac{t^{(\beta-2) i}}{\Gamma(1-(2-\beta) i)}, \quad t \longrightarrow \infty .
\end{aligned}
$$

The behaviour at zero follows from (3.4) and the fact that $E_{\gamma}(0)=1, \gamma>0$.

A similar result which has been proved in [8] and will be employed in Section 4.2 is the following lemma. 
LEMMA 3.3. Consider the integrodifferential equation

$$
\chi^{\prime}(t)=-\int_{0}^{t} b(t-s) \chi(s) d s, \quad 0<t, \chi(0)=\chi_{0} .
$$

Suppose that

(i) $b(t) \in C(0, \infty) \bigcap L_{1}(0,1)$,

(ii) $(-1)^{(j)} b^{(j)}(t) \geq 0, j=0,1,2, \ldots$,

(iii) $b(t) \not \equiv b\left(0_{+}\right)$.

Then,

$$
\chi^{(n)}(t)=o\left(t^{-n-1}\right), \quad t \rightarrow \infty, n=0,1,2 .
$$

Lemma 3.4 below is the result obtained by Bleistein and Handelsman [3]. The Mellin transform of a locally integrable function $\phi(t)$ on $(0, \infty)$ is defined by

$$
M[\phi ; z]=\int_{0}^{\infty} t^{z-1} \phi(t) d t
$$

when the integral converges.

LEMMA 3.4. Consider the integral

$$
I_{\eta}[\psi: \lambda]=\frac{\lambda^{\eta}}{\Gamma(\eta)} \int_{0}^{1}(1-\theta)^{\eta-1} \psi(\lambda \theta) d \theta, \quad 0<\eta<1, \lambda>0 .
$$

(i) Let $\psi(\theta)=O\left(\theta^{-a_{0}}\right), \theta \rightarrow 0$,

(ii) let $\psi(\theta)=O\left(\theta^{-a_{1}}\right), \theta \rightarrow \infty$, where $a_{0}$ and $a_{1}$ are constants such that $a_{0}<a_{1}$,

(iii) there exists a Mellin transform of $\psi$, denoted by $M[\psi ; 1]$ and is given by the integral

$$
M[\psi ; 1]=\int_{0}^{\infty} \psi(\theta) d \theta
$$

which is absolutely convergent and holomorphic in the strip $a_{0}<1<a_{1}$. Then, as $\lambda \rightarrow \infty, I_{\eta}$ has the following leading-order asymptotic representation:

$$
I_{\eta}[\psi: \lambda] \sim \frac{M[\psi ; 1] \lambda^{\eta-1}}{\Gamma(\eta)} .
$$

For more on Mellin transforms and its properties, see [12].

4. Heuristic analysis and formal solution. To start with, one assumes an asymptotic approximation

$$
y(t ; \varepsilon)=u(t ; \varepsilon)+\mu(\varepsilon) v\left(\frac{t}{\varepsilon^{\alpha}} ; \varepsilon\right), \quad \frac{t}{\varepsilon^{\alpha}}=\tau,
$$


where

$\alpha$ is a constant,

$$
\begin{aligned}
& u(t ; \varepsilon)=u_{0}(t)+o(1), \quad \varepsilon \longrightarrow 0, \\
& v(\tau ; \varepsilon)=v_{0}(\tau)+o(1), \quad \varepsilon \longrightarrow 0 .
\end{aligned}
$$

Then, the width $\left(\varepsilon^{\alpha}\right)$ of the initial layer and the magnitude $\mu(\varepsilon)$ of the solution in the layer are determined formally, assuming that $\alpha>0$. The substitution of (4.1a) into (1.1) yields

$$
\begin{aligned}
\varepsilon u^{\prime}(t ; \varepsilon)+\mu(\varepsilon) \varepsilon^{1-\alpha} v^{\prime}\left(\frac{t}{\varepsilon^{\alpha}} ; \varepsilon\right)= & g(t)+\frac{1}{\Gamma(1-\beta)} \int_{0}^{t} \frac{k(t, s)}{(t-s)^{\beta}} u(s ; \varepsilon) d s \\
& +\frac{\mu(\varepsilon)}{\Gamma(1-\beta)} \int_{0}^{t} \frac{k(t, s)}{(t-s)^{\beta}} v\left(\frac{s}{\varepsilon^{\alpha}} ; \varepsilon\right) d s .
\end{aligned}
$$

Expressing the above equation in terms of $\tau$, one obtains

$$
\begin{aligned}
\varepsilon u^{\prime}\left(\varepsilon^{\alpha} \tau ; \varepsilon\right)+\mu(\varepsilon) \varepsilon^{1-\alpha} v^{\prime}(\tau ; \varepsilon) \\
=g\left(\varepsilon^{\alpha} \tau\right)+\frac{\varepsilon^{\alpha(1-\beta)}}{\Gamma(1-\beta)} \int_{0}^{\tau} \frac{k\left(\varepsilon^{\alpha} \tau, \varepsilon^{\alpha} \sigma\right)}{(\tau-\sigma)^{\beta}} u(\sigma ; \varepsilon) d \sigma \\
\quad+\frac{\mu(\varepsilon) \varepsilon^{\alpha(1-\beta)}}{\Gamma(1-\beta)} \int_{0}^{\tau} \frac{k\left(\varepsilon^{\alpha} \tau, \varepsilon^{\alpha} \sigma\right)}{(\tau-\sigma)^{\beta}} v(\sigma ; \varepsilon) d \sigma .
\end{aligned}
$$

Using (4.1b), the equation above can similarly be written as

$$
\begin{aligned}
\varepsilon^{1-\alpha} \mu(\varepsilon) & v_{0}^{\prime}(\tau) \\
= & g(0)+\varepsilon^{\alpha} \tau g^{\prime}(0)+\frac{\varepsilon^{\alpha(1-\beta)}}{\Gamma(1-\beta)} \int_{0}^{\tau} \frac{k(0,0)}{(\tau-\sigma)^{\beta}} u_{0}\left(\varepsilon^{\alpha} \sigma\right) d \sigma \\
& +\frac{\mu(\varepsilon) \varepsilon^{\alpha(1-\beta)}}{\Gamma(1-\beta)} \int_{0}^{\tau} \frac{k(0,0)}{(\tau-\sigma)^{\beta}} v_{0}(\sigma) d \sigma+O\left(\varepsilon^{\alpha}\right)+O(\varepsilon), \quad \varepsilon \longrightarrow 0 .
\end{aligned}
$$

To balance the dominant terms, one has to distinguish the two cases: $g(0) \neq 0$ and $g(0)=0$. When $g(0) \neq 0$, one needs to choose

$$
\mu(\varepsilon)=\varepsilon^{-\alpha(1-\beta)}, \quad \alpha=\frac{1}{2-\beta} .
$$

And when $g(0)=0$, one chooses

$$
\mu(\varepsilon)=1, \quad \alpha=\frac{1}{2-\beta} .
$$


It then follows that $0<\beta<\alpha<1$ and therefore the singularly perturbed problem (1.1) has an initial layer width of order $O\left(\varepsilon^{\alpha}\right), \varepsilon \rightarrow 0$. The magnitude depends on whether $g(0)=0$, when it is of order $O(1)$, as $\varepsilon \rightarrow 0$, or $g(0) \neq 0$, when it is of order $O\left(\varepsilon^{-\alpha(1-\beta)}\right)$, as $\varepsilon \rightarrow 0$.

Note that for $\varepsilon>0, \varphi(\tau ; \varepsilon)=\varepsilon^{\alpha(1-\beta)} y\left(\varepsilon^{\alpha} \tau ; \varepsilon\right), \tau>0$, satisfies

$$
\begin{aligned}
\varphi^{\prime}(\tau ; \varepsilon)= & g\left(\varepsilon^{\alpha} \tau\right) \\
& +\frac{1}{\Gamma(1-\beta)} \int_{0}^{\tau} \frac{k\left(\varepsilon^{\alpha} \tau, \varepsilon^{\alpha} \sigma\right)}{(\tau-\sigma)^{\beta}} \varphi(\sigma, \varepsilon) d \sigma, \quad 0<\tau \leq \frac{T}{\varepsilon^{\alpha}}, \varphi(0 ; \varepsilon)=y_{0}(\varepsilon) .
\end{aligned}
$$

The limiting equations for $g(0) \neq 0$ and $g(0)=0$ obtained by letting $\varepsilon \rightarrow 0$ are, respectively,

$$
\begin{aligned}
& \varphi_{0}^{\prime}(\tau)=g(0)+\frac{1}{\Gamma(1-\beta)} \int_{0}^{\tau} \frac{k(0,0)}{(\tau-\sigma)^{\beta}} \varphi_{0}(\sigma) d \sigma, \quad \varphi_{0}(0)=\frac{\tilde{y}_{0}}{\xi(\varepsilon)} \\
& \varphi_{0}^{\prime}(\tau)=\frac{1}{\Gamma(1-\beta)} \int_{0}^{\tau} \frac{k(0,0)}{(\tau-\sigma)^{\beta}} \varphi_{0}(\sigma) d \sigma, \quad \varphi_{0}(0)=y_{0} .
\end{aligned}
$$

This leads to the approximation

$$
\frac{\tilde{y}_{0}}{\xi(\varepsilon)} E_{2-\beta}\left(k(0,0) \tau^{2-\beta}\right)+g(0) \int_{0}^{\tau} E_{2-\beta}\left(k(0,0) \sigma^{2-\beta}\right) d \sigma, \quad g(0) \neq 0
$$

or

$$
y_{0} E_{2-\beta}\left(k(0,0) \tau^{2-\beta}\right), \quad g(0)=0
$$

the solution in the initial layer region. While the case $k(0,0)=0$ does not give rise to an initial layer, $k(0,0)>0$ yields algebraic growth in the layer region and therefore it is of less interest.

Next, the formal approximate solution of (1.1) is derived. This is accomplished by deriving integral equations governing $u_{0}$ and $v_{0}$ in (4.1). As will be shown, these equations are much simpler to be analyzed than (1.1). The analysis presented in the derivation of the formal solution distinguishes the cases when $g(0) \neq 0$ and $g(0)=0$, due to their differences. The differences between the two cases are twofold. Not only is the form of the asymptotic expansion different, but also the initial layer correction solution can be constructed first in the case $g(0) \neq 0$ whereas the outer solution must be found first in the case $g(0)=0$. Hence, in what follows in this section and in Section 5, the two cases will be treated separately. 
4.1. Formal solution corresponding to the case when $g(0) \neq 0$. This is the case where the reduced equation, obtained by setting $\varepsilon=0$, does not have a solution, resulting in large magnitude of the solution in the initial layer. As shown below, due to this large magnitude, there is a nontrivial contribution to the outer solution from the integral (1.1) in the initial layer limit as $\varepsilon \rightarrow 0$ with $t>0$ fixed. Thus, in this section, (1.1) is considered with $y_{0}(\varepsilon)=\tilde{y}_{0} / \varepsilon^{\alpha(1-\beta)}$.

The form of (4.9a) suggests that one has to seek an approximate solution in the form of

$$
y(t ; \varepsilon):=y_{0}(t ; \varepsilon)=u_{0}(t)+\varepsilon^{-\alpha(1-\beta)} v_{0}\left(\frac{t}{\varepsilon^{\alpha}}\right), \quad \alpha=\frac{1}{2-\beta}
$$

Equations governing $v_{0}$ and $u_{0}$ will be derived assuming that

$$
v_{0}(\tau) \sim \frac{g(0) \tau^{\beta-1}}{\Gamma(\beta)}, \quad \tau \rightarrow \infty
$$

holds. Condition (4.11) will be proved, once the approximate solution is derived. Suppose $y_{0}(t ; \varepsilon)$ satisfies (1.1) approximately, with a residual $\rho(t ; \varepsilon)$, then

$$
\begin{aligned}
\varepsilon u_{0}^{\prime}(t)+v_{0}^{\prime}\left(\frac{t}{\varepsilon^{\alpha}}\right)= & g(t)+\frac{1}{\Gamma(1-\beta)} \int_{0}^{t} \frac{k(t, s)}{(t-s)^{\beta}} u_{0}(s) d s \\
& +\frac{\varepsilon^{-\alpha(1-\beta)}}{\Gamma(1-\beta)} \int_{0}^{t} \frac{k(t, s)}{(t-s)^{\beta}} v_{0}\left(\frac{s}{\varepsilon^{\alpha}}\right) d s-\rho(t ; \varepsilon) .
\end{aligned}
$$

Expressing (4.12) in terms of $\tau$ yields

$$
\begin{aligned}
\rho\left(\varepsilon^{\alpha} \tau ; \varepsilon\right)+\varepsilon u_{0}^{\prime}\left(\varepsilon^{\alpha} \tau\right)+v_{0}^{\prime}(\tau)= & g\left(\varepsilon^{\alpha} \tau\right)+\frac{\varepsilon^{\alpha(1-\beta)}}{\Gamma(1-\beta)} \int_{0}^{\tau} \frac{k\left(\varepsilon^{\alpha} \tau, \varepsilon^{\alpha} \sigma\right)}{(\tau-\sigma)^{\beta}} u_{0}\left(\varepsilon^{\alpha} \sigma\right) d \sigma \\
& +\frac{1}{\Gamma(1-\beta)} \int_{0}^{\tau} \frac{k\left(\varepsilon^{\alpha} \tau, \varepsilon^{\alpha} \sigma\right)}{(\tau-\sigma)^{\beta}} v_{0}(\sigma) d \sigma .
\end{aligned}
$$

This can equivalently be written as

$$
\begin{aligned}
\rho\left(\varepsilon^{\alpha} \tau ; \varepsilon\right)+\varepsilon u_{0}^{\prime}\left(\varepsilon^{\alpha} \tau\right)= & \left\{-v_{0}^{\prime}(\tau)+g(0)+\frac{1}{\Gamma(1-\beta)} \int_{0}^{\tau} \frac{k(0,0)}{(\tau-\sigma)^{\beta}} v_{0}(\sigma) d \sigma\right\} \\
& +g\left(\varepsilon^{\alpha} \boldsymbol{T}\right)-g(0) \\
& +\frac{1}{\Gamma(1-\beta)} \int_{0}^{\tau} \frac{k\left(\varepsilon^{\alpha} \boldsymbol{T}, \varepsilon^{\alpha} \sigma\right)-k(0,0)}{(\tau-\sigma)^{\beta}} v_{0}(\sigma) d \sigma \\
& +\frac{\varepsilon^{\alpha(1-\beta)}}{\Gamma(1-\beta)} \int_{0}^{\tau} \frac{k\left(\varepsilon^{\alpha} \boldsymbol{T}, \varepsilon^{\alpha} \sigma\right)}{(\tau-\sigma)^{\beta}} u_{0}\left(\varepsilon^{\alpha} \sigma\right) d \sigma .
\end{aligned}
$$


For $\varepsilon$ small, the above equation is the same as

$$
\begin{aligned}
\rho\left(\varepsilon^{\alpha} \tau ; \varepsilon\right)=\{ & -v_{0}^{\prime}(\tau)+g(0) \\
& \left.+\frac{1}{\Gamma(1-\beta)} \int_{0}^{\tau} \frac{k(0,0)}{(\tau-\sigma)^{\beta}} v_{0}(\sigma) d \sigma\right\}+O\left(\varepsilon^{\alpha}\right), \quad \varepsilon \longrightarrow 0 .
\end{aligned}
$$

If $\rho\left(\varepsilon^{\alpha} \tau ; \varepsilon\right)=o(1)$ as $\varepsilon \rightarrow 0$, we have for a fixed $\tau>0$, the leading-order inner equation,

$$
v_{0}^{\prime}(\tau)=g(0)+\frac{1}{\Gamma(1-\beta)} \int_{0}^{\tau} \frac{k(0,0)}{(\tau-\sigma)^{\beta}} v_{0}(\sigma) d \sigma, \quad \tau>0, v_{0}(0)=\tilde{y}_{0}
$$

To obtain the leading-order outer equation, express (4.16) in terms of $t=\varepsilon^{\alpha} \boldsymbol{T}$ and substitute into (4.12) giving

$$
\begin{aligned}
\rho(t ; \varepsilon)+\varepsilon u_{0}^{\prime}(t)= & g(t)-g(0)+\frac{1}{\Gamma(1-\beta)} \int_{0}^{t} \frac{k(t, s)}{(t-s)^{\beta}} u_{0}(s) d s \\
& +\frac{\varepsilon^{-\alpha(1-\beta)}}{\Gamma(1-\beta)} \int_{0}^{t} \frac{k(t, s)-k(0,0)}{(t-s)^{\beta}} v_{0}\left(\frac{s}{\varepsilon^{\alpha}}\right) d s .
\end{aligned}
$$

The dominated convergence theorem and (4.11) imply that, as $\varepsilon \rightarrow 0$,

$$
\frac{\varepsilon^{-\alpha(1-\beta)}}{\Gamma(1-\beta)} \int_{0}^{t} \frac{k(t, s)-k(0,0)}{(t-s)^{\beta}} v_{0}\left(\frac{s}{\varepsilon^{\alpha}}\right) d s \longrightarrow \frac{g(0)}{\Gamma(\beta) \Gamma(1-\beta)} \int_{0}^{t} \frac{k(t, s)-k(0,0)}{(t-s)^{\beta} s^{1-\beta}} d s .
$$

Thus, if $\rho(t ; \varepsilon)=o(1)$ as $\varepsilon \rightarrow 0$ for a fixed $t>0,(4.17)$ implies that the leadingorder outer solution satisfies

$$
\begin{aligned}
0= & g(t)-g(0)+\frac{g(0)}{\Gamma(\beta) \Gamma(1-\beta)} \int_{0}^{t} \frac{k(t, s)-k(0,0)}{(t-s)^{\beta} s^{1-\beta}} d s \\
& +\frac{1}{\Gamma(1-\beta)} \int_{0}^{t} \frac{k(t, s)}{(t-s)^{\beta}} u_{0}(s) d s .
\end{aligned}
$$

If $v_{0}(\tau)$ and $u_{0}(t)$ satisfy (4.16) and (4.19), respectively, then $\rho(t ; \varepsilon)$ obeys

$$
\begin{aligned}
\rho(t ; \varepsilon)= & -\varepsilon u_{0}^{\prime}(t)+\frac{\varepsilon^{-\alpha(1-\beta)}}{\Gamma(1-\beta)} \int_{0}^{t} \frac{k(t, s)-k(0,0)}{(t-s)^{\beta}} v_{0}\left(\frac{s}{\varepsilon^{\alpha}}\right) d s \\
& -\frac{g(0)}{\Gamma(\beta) \Gamma(1-\beta)} \int_{0}^{t} \frac{k(t, s)-k(0,0)}{(t-s)^{\beta} s^{1-\beta}} d s .
\end{aligned}
$$


4.2. Formal solution corresponding to the case when $g(0)=0$. In this case, the reduced equation has a solution. For clarity, consider cases similar to the model described by (2.3), let

$$
k(t, s)=-1, \quad 0 \leq s \leq t \leq T .
$$

However, a similar analysis can be applied to equations with more general kernels as in (1.1). Thus, consider the singularly perturbed equation

$$
\varepsilon y^{\prime}(t)=g(t)-\frac{1}{\Gamma(1-\beta)} \int_{0}^{t}(t-s)^{-\beta} y(s) d s, \quad y(0)=y_{0}, \quad g(0)=0 .
$$

The form of (4.9b) suggests the following definition:

$$
y(t ; \varepsilon):=y_{0}(t ; \varepsilon)=u_{0}(t)+v_{0}\left(\frac{t}{\varepsilon^{\alpha}}\right), \quad \alpha=\frac{1}{2-\beta},
$$

for the formal approximate solution. The following will be assumed during the derivation of the approximate solution,

$$
\begin{gathered}
v_{0}(\tau)=O(1), \quad \tau \longrightarrow 0, \\
\lim _{\tau \rightarrow \infty} v_{0}^{\prime}(\tau)=0, \\
v_{0}(\tau) \sim \frac{v_{0}}{\Gamma(\beta-1)} \tau^{\beta-2}, \quad \tau \longrightarrow \infty .
\end{gathered}
$$

In a similar manner as in Section 4.1 , it is assumed that $y_{0}(t ; \varepsilon)$ satisfies (1.1) approximately with a residual $\rho(t ; \varepsilon)$, so that

$$
\begin{aligned}
\rho(t ; \varepsilon)+\varepsilon u_{0}^{\prime}(t)+\varepsilon^{1-\alpha} v_{0}^{\prime}\left(\frac{t}{\varepsilon^{\alpha}}\right)= & g(t)-\frac{1}{\Gamma(1-\beta)} \int_{0}^{t}(t-s)^{-\beta} u_{0}(s) d s \\
& -\frac{1}{\Gamma(1-\beta)} \int_{0}^{t}(t-s)^{-\beta} v_{0}\left(\frac{s}{\varepsilon^{\alpha}}\right) d s .
\end{aligned}
$$

Equation (4.25) can equivalently be written as

$$
\begin{aligned}
\rho(t ; \varepsilon)+\varepsilon u_{0}^{\prime}(t)+\varepsilon^{1-\alpha} v_{0}^{\prime}\left(\frac{t}{\varepsilon^{\alpha}}\right)= & g(t)-\frac{1}{\Gamma(1-\beta)} \int_{0}^{t}(t-s)^{-\beta} u_{0}(s) d s \\
& -\frac{t^{1-\beta}}{\Gamma(1-\beta)} \int_{0}^{1}(1-s)^{-\beta} v_{0}\left(\frac{t s}{\varepsilon^{\alpha}}\right) d s .
\end{aligned}
$$


If (4.24a) and (4.24c) are true, then conditions of Lemma 3.4 are satisfied and therefore

$$
\begin{aligned}
& \frac{\left(t / \varepsilon^{\alpha}\right)^{1-\beta}}{\Gamma(1-\beta)} \int_{0}^{1}(1-s)^{-\beta} v_{0}\left(\frac{t s}{\varepsilon^{\alpha}}\right) d s \\
& \quad=\varepsilon^{\alpha \beta} \frac{M\left[v_{0} ; 1\right] t^{-\beta}}{\Gamma(1-\beta)}, \quad \text { as } \varepsilon \longrightarrow 0, t>0,
\end{aligned}
$$

where $M\left[v_{0} ; 1\right]$ is the Mellin transform of $v_{0}$. Then, one writes (4.26) as

$$
\begin{aligned}
\rho(t ; \varepsilon)+\varepsilon^{1-\alpha} v_{0}^{\prime}\left(\frac{t}{\varepsilon^{\alpha}}\right)= & g(t)-\frac{1}{\Gamma(1-\beta)} \int_{0}^{t}(t-s)^{-\beta} u_{0}(s) d s \\
& +O\left(\varepsilon^{\alpha}\right)+O(\varepsilon), \quad \varepsilon \longrightarrow 0 .
\end{aligned}
$$

If $\rho(t ; \varepsilon)=o(1), \varepsilon \rightarrow 0$, then by fixing $t>0$ in (4.26) and letting $\varepsilon \rightarrow 0$, using (4.24b), the governing outer equation becomes

$$
0=g(t)-\frac{1}{\Gamma(1-\beta)} \int_{0}^{t}(t-s)^{-\beta} u_{0}(s) d s
$$

To derive the inner layer equation, substitute (4.29) into (4.25) and express all terms in terms of $\tau=t / \varepsilon^{\alpha}$. This yields

$$
\begin{aligned}
-\rho\left(\varepsilon^{\alpha} \tau ; \varepsilon\right)= & \varepsilon u_{0}^{\prime}\left(\varepsilon^{\alpha} \tau\right)+\varepsilon^{\alpha(1-\beta)} v_{0}^{\prime}(\tau) \\
& +\frac{\varepsilon^{\alpha(1-\beta)}}{\Gamma(1-\beta)} \int_{0}^{\tau}(\tau-\sigma)^{-\beta} v_{0}(\sigma) d \sigma .
\end{aligned}
$$

Multiplying throughout the above equation by $\varepsilon^{-\alpha(1-\beta)}$ gives

$$
-\varepsilon^{-\alpha(1-\beta)} \rho\left(\varepsilon^{\alpha} \tau ; \varepsilon\right)=v_{0}^{\prime}(\tau)+\frac{1}{\Gamma(1-\beta)} \int_{0}^{\tau}(\tau-\sigma)^{-\beta} v_{0}(\sigma) d \sigma+O\left(\varepsilon^{\alpha}\right)
$$

Then assuming that $\varepsilon^{-\alpha(1-\beta)} \rho\left(\varepsilon^{\alpha} \tau ; \varepsilon\right)=O\left(\varepsilon^{\alpha}\right)$ as $\varepsilon \rightarrow 0$, fixing $\tau>0$, and letting $\varepsilon \rightarrow 0$, the desired equation becomes

$$
v_{0}^{\prime}(\tau)=\frac{-1}{\Gamma(1-\beta)} \int_{0}^{\tau}(\tau-\sigma)^{-\beta} v_{0}(\sigma) d \sigma, \quad v_{0}(0)=y_{0}-u_{0}(0)
$$

If $u_{0}(t)$ and $v_{0}(\tau)$ satisfy (4.29) and (4.32), respectively, then it follows from (4.25) that the residual function satisfies

$$
\rho(t ; \varepsilon)=-\varepsilon u_{0}^{\prime}(t) .
$$


5. Properties of the formal solution. It is shown here that the components of the derived formal solution, $v_{0}(\tau)$ and $u_{0}(t)$, have the required properties (to match those of $y(t ; \varepsilon), \varepsilon>0$ ) and those properties assumed in their derivation. It is also shown that $\rho(t ; \varepsilon)=O(\varepsilon), \varepsilon \rightarrow 0$, uniformly for all $0 \leq t \leq T$. The cases $g(0) \neq 0$ and $g(0)=0$ are again treated separately.

5.1. Properties of the formal solution for case when $g(0) \neq 0$. The standard theory of Volterra integral equations and Lemma 3.2 justify the following result.

Proposition 5.1. Suppose that $\left(H_{\beta}\right),\left(H_{k}\right)$, and $\left(H_{g}\right)$ hold. Then, (4.16) has a continuous solution for all $\tau \geq 0$. Moreover, $v_{0}(\tau)$ satisfies (4.11).

Proposition 5.2 follows the results on Abel integral equation by Hille and Tamarkin [9] and by Gorenflo and Vessella [5]. The proof is therefore omitted.

Proposition 5.2. Suppose that $\left(H_{\beta}\right),\left(H_{k}\right)$, and $\left(H_{g}\right)$ hold. Then, (4.19) has a unique solution, $u_{0}(t) \in C^{1}[0, T]$.

Proposition 5.3. Suppose that $\rho(t ; \varepsilon)$ satisfies (4.20), then there exists a constant $C_{0}$ independent of $\varepsilon$ and $\varepsilon_{0}$ such that

$$
|\rho(t ; \varepsilon)| \leq C_{0} \varepsilon
$$

for all $0 \leq t \leq T$ and $0<\varepsilon \leq \varepsilon_{0}$.

Proof. The proof follows from (4.20), the dominated convergence theorem, and Propositions 5.1 and 5.2 with

$$
C_{0}=\sup _{0 \leq t \leq T}\left|u_{0}^{\prime}(t)\right|
$$

5.2. Properties of the formal solution for case when $g(0)=0$. A similar result to Proposition 5.2 is the following proposition.

Proposition 5.4. Suppose that $\left(H_{\beta}\right),\left(H_{k}\right)$, and $\left(H_{g}\right)$ hold. Then, (4.29) has a unique continuously differentiable solution $u_{0}(t)$ for all $0 \leq t \leq T$.

Proposition 5.5. Suppose that $\left(H_{\beta}\right),\left(H_{k}\right)$, and $\left(H_{g}\right)$ hold. Then, (4.32) has a continuous solution for all $\tau \geq 0$. Moreover, $v_{0}(\tau)$ satisfies (4.24).

Proof. The proof follows from the application of Lemmas 3.2 and 3.3.

Proposition 5.4 and (4.33) imply that the following proposition holds.

Proposition 5.6. Suppose that $\rho(t ; \varepsilon)$ satisfies (4.33), then there exists a constant $C_{1}$ independent of $\varepsilon$ and $\varepsilon_{0}$ such that

$$
|\rho(t ; \varepsilon)| \leq C_{1} \varepsilon
$$

for all $0 \leq t \leq T$ and $0<\varepsilon \leq \varepsilon_{0}$. 
6. Proof of asymptotic correctness. It is established in this section that the formal approximate solution, $y_{0}(t ; \varepsilon)$, given in $(4.1)$, is an asymptotic solution. The proof incorporates both cases: $g(0) \neq 0$ and $g(0)=0$. The main result in this paper is presented in the following theorem.

THeOrem 6.1. Suppose that $\left(H_{\beta}\right),\left(H_{k}\right)$, and $\left(H_{g}\right)$ are satisfied. Suppose also that Propositions 5.3 and 5.6 hold. Let $y(t ; \varepsilon) \in C[0, T], \varepsilon>0$, be the exact solution of (1.1). Then, (1.1) has an asymptotic solution $y_{0}(t ; \varepsilon) \in C[0, T]$ with the property that there exist positive constants $C^{\star}$ and $\varepsilon_{0}$ such that

$$
\left|y(t ; \varepsilon)-y_{0}(t ; \varepsilon)\right| \leq C^{\star} \varepsilon^{\alpha},
$$

uniformly for all $0 \leq t \leq T$ and all $0<\varepsilon \leq \varepsilon_{0}$.

It is convenient to introduce $r(t ; \varepsilon)=y(t ; \varepsilon)-y_{0}(t ; \varepsilon)$ which satisfies the equation

$$
\varepsilon r^{\prime}(t ; \varepsilon)=\rho(t ; \varepsilon)+\frac{1}{\Gamma(1-\beta)} \int_{0}^{t} \frac{k(t, s)}{(t-s)^{\beta}} r(s ; \varepsilon) d s, \quad 0<t \leq T, r(0 ; \varepsilon)=0 .
$$

Proof. The standard theory (see, e.g., [6]) of linear Volterra integrodifferential equations of the second kind ensures that for each $0<\varepsilon<\varepsilon_{0}$, (6.2) has a continuous solution $r(t ; \varepsilon)$ on $[0, T]$ given by

$$
r(t ; \varepsilon)=\frac{1}{\varepsilon} \int_{0}^{t} x(t, s ; \varepsilon) \rho(s ; \varepsilon) d s, \quad 0 \leq t \leq T,
$$

where $\chi(t, s ; \varepsilon)$ is the differential resolvent which satisfies

$$
\varepsilon \partial_{1} \chi(t, s ; \varepsilon)=\frac{1}{\Gamma(1-\beta)} \int_{s}^{t} \frac{k(t, \sigma)}{(t-\sigma)^{\beta}} \chi(\sigma, s ; \varepsilon) d \sigma, \quad \chi(t, t ; \varepsilon)=1,
$$

on $0 \leq s \leq t \leq T$. Again, the standard theory of Volterra equations ensures the existence, continuity, and uniqueness of $\chi(t, s ; \varepsilon)$ for $0<\varepsilon<\varepsilon_{0}$, refer to $[6,7,11]$. Following Propositions 5.3 and 5.6 to prove the theorem, one needs to show that there exists a positive constant $C_{2}$, which does not depend on $\varepsilon$ such that

$$
\int_{0}^{t}|\chi(t, s ; \varepsilon)| d s \leq C_{2} \varepsilon^{\alpha}
$$

for all $0 \leq t \leq T$ and all $0<\varepsilon \leq \varepsilon_{0}$.

To arrive at this inequality, integrate both sides of (6.4) with respect to $t$ giving

$$
\chi(t, s ; \varepsilon)=1+\frac{1}{\varepsilon \Gamma(2-\beta)} \int_{s}^{t} h(t, \sigma)(t-\sigma)^{1-\beta} \chi(\sigma, s ; \varepsilon) d \sigma
$$


where

$$
h(t, s)=(1-\beta) \int_{0}^{1} \frac{k(s+(t-s) \sigma, s)}{\sigma^{\beta}} d \sigma .
$$

Thus, $h(t, s)$ is a $C^{2}$ function on $0 \leq s \leq t \leq T$ and $h(t, t)=-1$ for all $0 \leq t \leq T$. It can be shown that there exists a positive constant $\varrho$ such that

$$
h(t, s) \leq-\varrho, \quad 0 \leq s \leq t \leq T .
$$

This therefore implies that

$$
0 \leq \chi(t, s ; \varepsilon) \leq 1, \quad \forall 0 \leq s \leq t \leq T, \forall 0<\varepsilon \leq \varepsilon_{0} .
$$

Thus, (6.6) can be converted into

$$
\chi(t, s ; \varepsilon) \leq 1-\frac{\varrho}{\varepsilon \Gamma(2-\beta)} \int_{s}^{t}(t-\sigma)^{1-\beta} \chi(\sigma, s ; \varepsilon) d \sigma
$$

If $t$ is replaced by $t+s$, the above inequality becomes

$$
\chi(t+s, s ; \varepsilon) \leq 1-\frac{\varrho}{\varepsilon \Gamma(2-\beta)} \int_{s}^{t+s}(t+s-\sigma)^{1-\beta} \chi(\sigma, s ; \varepsilon) d \sigma
$$

or equivalently,

$$
\chi(t+s, s ; \varepsilon) \leq 1-\frac{\varrho}{\varepsilon} J^{2-\beta} \chi(t+s, s ; \varepsilon) .
$$

In this form, $s$ is simply a parameter and if the role of this parameter is suppressed, one writes (6.10) as

$$
\tilde{\chi}(t ; \varepsilon) \leq 1-\frac{\varrho}{\varepsilon} J^{2-\beta} \tilde{\chi}(t ; \varepsilon)
$$

with $\tilde{\chi}(t ; \varepsilon):=\chi(t, s ; \varepsilon)$, for all $0 \leq s \leq t \leq T$ and all $0<\varepsilon \leq \varepsilon_{0}$.

To this point, comparison theorems are employed and one deals with

$$
\phi(t ; \varepsilon)=1-\frac{\varrho}{\varepsilon} J^{2-\beta} \phi(t ; \varepsilon),
$$

where

$$
0 \leq \tilde{X}(t ; \varepsilon) \leq \phi(t ; \varepsilon) \leq 1, \quad \forall 0 \leq t \leq T, \forall 0<\varepsilon \leq \varepsilon_{0} .
$$


Therefore, to accomplish the proof, it suffices to show that there exists a positive constant, $C_{3}$ say, which does not depend on $\varepsilon$ such that

$$
\int_{0}^{t} \phi(\sigma ; \varepsilon) d \sigma \leq C_{3} \varepsilon^{\alpha}
$$

uniformly for all $0 \leq t \leq T$ and all $0<\varepsilon \leq \varepsilon_{0}$.

Now, consider solving (6.14) using the Laplace transform method. Let $\Phi(s ; \varepsilon)$, $s \in \mathbb{C}$, denote the Laplace transform of $\phi(t ; \varepsilon)$, then

$$
\Phi(s ; \varepsilon)=\frac{s^{1-\beta}}{s^{2-\beta}+\varrho / \varepsilon} .
$$

Applying the inverse Laplace transformation in (6.17) and using the pair in (3.8), one obtains

$$
\phi(t ; \varepsilon)=E_{2-\beta}\left(-\frac{\varrho}{\varepsilon} t^{2-\beta}\right)
$$

the Mittag-Leffler function of order $2-\beta$. By definition,

$$
E_{2-\beta}\left(-\frac{\varrho}{\varepsilon} t^{2-\beta}\right)=\sum_{n=0}^{\infty} \frac{(-\varrho / \varepsilon)^{n} t^{n(2-\beta)}}{\Gamma(n(2-\beta)+1)}, \quad t>0, E_{2-\beta}(0)=1
$$

Since the interest here is in the boundedness of this function for small values of $\varepsilon$, one writes

$$
\phi(t ; \varepsilon)=\frac{1}{2 \pi i} \int_{\tilde{C}} \frac{s^{1-\beta}}{s^{2-\beta}+\varrho / \varepsilon} e^{s t} d s
$$

where $\tilde{C}$ consists of a line $\operatorname{Re} s=\eta$, for some $\eta>(\varrho / \varepsilon)^{\alpha}$.

Properties of the function $e_{\gamma}(t ; \lambda):=E_{\gamma}\left(-\lambda t^{\gamma}\right), 0<\gamma \leq 3$, defined by

$$
e_{\sigma}(t ; \lambda)=\frac{1}{2 \pi i} \int_{\tilde{C}} \frac{s^{\gamma-1}}{s^{\gamma}+\lambda} e^{s t} d s
$$

have been discussed in [4]. Following a similar analysis, it can be shown that, by bending the path of integration in $(6.20), \phi(t ; \varepsilon)$ can be written as a sum of two functions

$$
\phi(t ; \varepsilon)=\varphi(t ; \varepsilon)+\psi(t ; \varepsilon), \quad 0 \leq t \leq T, 0<\varepsilon \leq \varepsilon_{0},
$$


where $-\varphi$ is completely monotone, tends to zero (algebraically) as $t$ tends to infinity and $\psi$ is of oscillatory character with an exponentially decaying magnitude. On performing complex integration (details omitted), one obtains

$$
\begin{aligned}
& \varphi(t ; \varepsilon)=\frac{\varrho / \varepsilon \sin (\pi / \alpha)}{\pi} \int_{0}^{\infty} \frac{e^{-\sigma t} \sigma^{1-\beta}}{\sigma^{2(2-\beta)}+2(\varrho / \varepsilon) \cos (\pi / \alpha) \sigma^{2-\beta}+\varrho^{2} / \varepsilon^{2}} d \sigma, \\
& \psi(t ; \varepsilon)=2 \alpha \exp \left(\left(\frac{\varrho}{\varepsilon}\right)^{\alpha} \cos (\alpha \pi) t\right) \cos (t \sin (\alpha \pi)), \quad 0<\beta<\alpha<1 .
\end{aligned}
$$

If one puts $\sin (\pi / \alpha)=v_{0}, \cos (\pi / \alpha)=v_{1}, \cos (\alpha \pi)=v_{2}$, and $\sin (\alpha \pi)=v_{3}$, then $v_{0}<0, v_{2}<0, v_{3}>0, v_{1} \in(-1,1)$, and

$$
\phi(t ; \varepsilon)=\frac{\varrho u_{0}}{\pi} \int_{0}^{\infty} \frac{e^{-\sigma t / \varepsilon^{\alpha}} \sigma^{1-\beta}}{\sigma^{2(2-\beta)}+2 \varrho v_{1} \sigma^{2-\beta}+\varrho^{2}} d \sigma+2 \alpha e^{u_{2}(\varrho / \varepsilon)^{\alpha} t} \cos \left(u_{3} t\right)
$$

such that

$$
\int_{0}^{\infty} \frac{\sigma^{1-\beta}}{\sigma^{2(2-\beta)}+2 \varrho U_{1} \sigma^{2-\beta}+\varrho^{2}} d \sigma=\frac{(1-2 \alpha) \pi}{\varrho v_{0}}
$$

The fact that the denominator of the integrand in (6.24) is always positive implies that $-\varphi$ is completely monotone. The function $\psi$ has an exponentially decaying amplitude. The relation in (6.25) follows by putting $t=0$ in (6.14) or in (6.19). The required result then follows from (6.24) as

$$
0 \leq \int_{0}^{t} \phi(s ; \varepsilon) d s \leq \frac{4 \alpha \varepsilon^{\alpha}}{\left|v_{2}\right| \varrho}, \quad 0 \leq t \leq T, 0<\varepsilon \leq \varepsilon_{0} .
$$

This implies that

$$
|r(t ; \varepsilon)| \leq \frac{4 C \alpha \varepsilon^{\alpha}}{\left|v_{2}\right| \varrho}, \quad 0 \leq t \leq T, 0<\varepsilon \leq \varepsilon_{0}
$$

where $C$ is a positive constant which depends on $T$.

7. Example. In this section, the methodologies developed in the previous sections are employed to find an approximate asymptotic solution $C(x, t)$ of 
(2.2). In particular, the analysis in Section 4.2 is used to derive the formal asymptotic solution, $y_{0}(t ; \varepsilon)$, of (2.3) and then demonstrate that it is asymptotically valid.

The exact solution of (2.3) obtained using the application of Laplace transforms is

$$
\begin{aligned}
y(t ; \varepsilon)= & \frac{1}{3} f(t)-\frac{f(0)}{3} e^{t / \varepsilon^{2 / 3}} \operatorname{erfc}\left\{\frac{\sqrt{t}}{\varepsilon^{1 / 3}}\right\} \\
& -\frac{1}{3} \int_{0}^{t} \exp \left[\frac{(t-s)}{\varepsilon^{2 / 3}}\right] \operatorname{erfc}\left[\frac{\sqrt{t-s}}{\varepsilon^{1 / 3}}\right] f^{\prime}(s) d s \\
& +\frac{2 \varepsilon^{-1 / 3}}{\sqrt{3 \pi}} \int_{0}^{t} \frac{f(t-s)}{s^{1 / 2}} \int_{0}^{\infty} \sigma \exp \left[-\sigma^{2}+\frac{\sigma \sqrt{s}}{\varepsilon^{1 / 3}}\right] \sin \left(\frac{\sqrt{3 s} \sigma}{\varepsilon^{1 / 3}}\right) d \sigma d s \\
& -\frac{2 \varepsilon^{-1 / 3}}{3 \sqrt{\pi}} \int_{0}^{t} \frac{f(t-s)}{s^{1 / 2}} \int_{0}^{\infty} \sigma \exp \left[-\sigma^{2}+\frac{\sigma \sqrt{s}}{\varepsilon^{1 / 3}}\right] \cos \left(\frac{\sqrt{3 s} \sigma}{\varepsilon^{1 / 3}}\right) d \sigma d s .
\end{aligned}
$$

The presence of terms which cannot be expanded in a series of nonnegative powers of $\varepsilon$ in a neighborhood of $\varepsilon=0$ shows that the solution of problem (2.2) depends on $\varepsilon$ in a singular manner.

However, the approximate solution obtained using the analysis in Section 4.2 is

$$
\begin{aligned}
y_{0}(t ; \varepsilon)=f(t)-\frac{f(0)}{3}\left\{e^{t / \varepsilon^{2 / 3}} \operatorname{erfc}\left(\frac{\sqrt{t}}{\varepsilon^{1 / 3}}\right)\right. & \\
+ & \left.\frac{4}{\sqrt{\pi}} \int_{0}^{\infty} \exp \left[-\sigma^{2}+\frac{\sigma \sqrt{t}}{\varepsilon^{1 / 3}}\right] \cos \left(\frac{\sqrt{3 t} \sigma}{\varepsilon^{1 / 3}}\right) d \sigma\right\},
\end{aligned}
$$

where

$$
u_{0}(t)=f(t)
$$

is the outer solution and

$$
v_{0}(\tau)=-\frac{f(0)}{3}\left\{e^{\tau} \operatorname{erfc}(\sqrt{\tau})+\frac{4}{\sqrt{\pi}} \int_{0}^{\infty} e^{-\sigma^{2}+\sigma \sqrt{\tau}} \cos (\sqrt{3 \tau} \sigma) d \sigma\right\}
$$

is the inner layer correction solution. It can be shown that both $u_{0}(t)$ and $v_{0}(\tau)$ satisfy the conditions that led to their derivation. 
To see that the remainder term is uniformly of order $O\left(\varepsilon^{2 / 3}\right)$, consider the difference $r(t ; \varepsilon)=y(t ; \varepsilon)-y_{0}(t ; \varepsilon)$;

$$
\begin{aligned}
r(t ; \varepsilon)= & y(t ; \varepsilon)-y_{0}(t ; \varepsilon) \\
= & -\frac{2}{3} f(t)-\frac{1}{3} \int_{0}^{t} \exp \left[\frac{(t-s)}{\varepsilon^{2 / 3}}\right] \operatorname{erfc}\left[\frac{\sqrt{t-s}}{\varepsilon^{1 / 3}}\right] f^{\prime}(s) d s \\
& +\frac{4 f(0)}{3} \int_{0}^{\infty} \exp \left[-\sigma^{2}+\frac{\sigma \sqrt{t}}{\varepsilon^{1 / 3}}\right] \cos \left(\frac{\sqrt{3 t} \sigma}{\varepsilon^{1 / 3}}\right) d \sigma \\
& +\frac{2 \varepsilon^{-1 / 3}}{\sqrt{3 \pi}} \int_{0}^{t} \frac{f(t-s)}{s^{1 / 2}} \int_{0}^{\infty} \sigma \exp \left[-\sigma^{2}+\frac{\sigma \sqrt{s}}{\varepsilon^{1 / 3}}\right] \sin \left(\frac{\sqrt{3 s} \sigma}{\varepsilon^{1 / 3}}\right) d \sigma d s \\
& -\frac{2 \varepsilon^{-1 / 3}}{3 \sqrt{\pi}} \int_{0}^{t} \frac{f(t-s)}{s^{1 / 2}} \int_{0}^{\infty} \sigma \exp \left[-\sigma^{2}+\frac{\sigma \sqrt{s}}{\varepsilon^{1 / 3}}\right] \cos \left(\frac{\sqrt{3 s} \sigma}{\varepsilon^{1 / 3}}\right) d \sigma d s .
\end{aligned}
$$

Integrating the integrals by parts, twice, both with respect to $s$ and $\sigma$, one obtains

$$
\begin{aligned}
r(t ; \varepsilon)= & -\varepsilon^{2 / 3} \frac{f^{\prime}(0)}{3} e^{t / \varepsilon^{2 / 3}} \operatorname{erfc}\left\{\frac{\sqrt{t}}{\varepsilon^{1 / 3}}\right\} \\
& -\frac{\varepsilon^{2 / 3}}{3} \int_{0}^{t} \exp \left[\frac{(t-s)}{\varepsilon^{2 / 3}}\right] \operatorname{erfc}\left[\frac{\sqrt{t-s}}{\varepsilon^{1 / 3}}\right] f^{\prime \prime}(s) d s \\
& +\frac{2 \varepsilon^{2 / 3} f^{\prime}(0)}{3 \sqrt{\pi}} \int_{0}^{\infty} \exp \left[-\sigma^{2}+\frac{\sigma \sqrt{t}}{\varepsilon^{1 / 3}}\right] \\
& \times\left\{\cos \left(\frac{\sqrt{3 t} \sigma}{\varepsilon^{1 / 3}}\right)-\sqrt{3} \sin \left(\frac{\sqrt{3 t} \sigma}{\varepsilon^{1 / 3}}\right)\right\} d \sigma \\
& -\frac{\varepsilon^{2 / 3}}{3 \sqrt{\pi}} \int_{0}^{t} \frac{f^{\prime \prime}(t-s)}{s^{1 / 2}} \int_{0}^{\infty} \exp \left[-\sigma^{2}+\frac{\sigma \sqrt{s}}{\varepsilon^{1 / 3}}\right] \\
& \times\left\{\cos \left(\frac{\sqrt{3 s} \sigma}{\varepsilon^{1 / 3}}\right)-\sqrt{3} \sin \left(\frac{\sqrt{3 s} \sigma}{\varepsilon^{1 / 3}}\right)\right\} d \sigma d s .
\end{aligned}
$$

Since

$$
\int_{0}^{\infty} \exp \left[-\sigma^{2}+\frac{\sigma \sqrt{t}}{\varepsilon^{1 / 3}}\right]\left\{\cos \left(\frac{\sqrt{3 t} \sigma}{\varepsilon^{1 / 3}}\right)-\sqrt{3} \sin \left(\frac{\sqrt{3 t} \sigma}{\varepsilon^{1 / 3}}\right)\right\} d \sigma<\infty, \quad 0 \leq t \leq T,
$$

it follows that

$$
\left|y(t ; \varepsilon)-y_{0}(t ; \varepsilon)\right|=O\left(\varepsilon^{2 / 3}\right), \quad \varepsilon \longrightarrow 0,
$$


uniformly for all $0 \leq t \leq T$. Note that the first term in (7.6) decays algebraically as $t$ tends to infinity while the remaining terms are of oscillatory character. This example verifies the possibility of weakening assumption $\mathrm{H}_{\mathfrak{g}}$.

8. Concluding remarks. The most interesting part of this investigation is the application of the properties of the Mittag-Leffler function in proving that the approximate solution is asymptotically valid. It is also interesting to see that, in this case where the region of nonuniformity in the solution is thicker (compared to similar equations with smooth kernels), the approximate solution converges slower to the exact solution than it does for solutions with smooth kernels.

The asymptotic solution constructed in Section 4 is limited only to the leading-order. It is of great interest to develop a methodology which yields asymptotic solution to all orders, as has been done for equations with continuous kernels.

The nonlinear version of (1.1) is currently being investigated by the author. There still, however, much work to be done in this particular area of singular perturbation.

ACKNOwLEDGments. This work has been supported by the Claude Harris Leon Foundation. The author wishes to thank Prof. Igor Barashenkov for hosting her, and the referees for their valuable comments.

\section{REFERENCES}

[1] J. S. Angell and W. E. Olmstead, Singularly perturbed Volterra integral equations. II, SIAM J. Appl. Math. 47 (1987), no. 6, 1150-1162.

[2] A. Bijura, Singularly perturbed Volterra integral equations with weakly singular kernels, Int. J. Math. Math. Sci. 30 (2002), no. 3, 129-143.

[3] N. Bleistein and R. A. Handelsman, Asymptotic Expansions of Integrals, Holt, Rinehart and Winston, New York, 1975.

[4] R. Gorenflo and F. Mainardi, Fractional calculus: integral and differential equations of fractional order, Fractals and Fractional Calculus in Continuum Mechanics (Udine, 1996) (A. Carpinteri and F. Mainardi, eds.), CISM Courses and Lectures, vol. 378, Springer, Vienna, 1997, pp. 223-276.

[5] R. Gorenflo and S. Vessella, Abel Integral Equations. Analysis and Applications, Lecture Notes in Mathematics, vol. 1461, Springer-Verlag, Berlin, 1991.

[6] G. Gripenberg, S.-O. Londen, and O. Staffans, Volterra integral and functional equations, Encyclopedia of Mathematics and Its Applications, vol. 34, Cambridge University Press, Cambridge, 1990.

[7] S. I. Grossman and R. K. Miller, Perturbation theory for Volterra integrodifferential systems, J. Differential Equations 8 (1970), 457-474.

[8] K. B. Hannsgen, A Volterra equation with completely monotonic convolution kernel, J. Math. Anal. Appl. 31 (1970), 459-471.

[9] E. Hille and J. D. Tamarkin, On the theory of linear integral equations, Ann. of Math. 31 (1930), 479-528.

[10] W. J. Hrusa, J. A. Nohel, and M. Renardy, Initial value problems in viscoelasticity, Appl. Mech. Rev. 41 (1988), no. 10, 371-378. 
[11] R. K. Miller, Nonlinear Volterra Integral Equations, Mathematics Lecture Note Series, W. A. Benjamin, California, 1971.

[12] R. B. Paris and D. Kaminski, Asymptotics and Mellin-Barnes Integrals, Encyclopedia of Mathematics and Its Applications, vol. 85, Cambridge University Press, Cambridge, 2001.

[13] M. Renardy, W. J. Hrusa, and J. A. Nohel, Mathematical Problems in Viscoelasticity, Pitman Monographs and Surveys in Pure and Applied Mathematics, vol. 35, John Wiley \& Sons, New York, 1987.

Angelina M. Bijura: Department of Mathematics, University of Dar Es Salaam, P.O. Box 35062, Dar Es Salaam, Tanzania

Current address: Department of Mathematics and Applied Mathematics, University of Cape Town, Cape Town, South Africa

E-mail address: abijura@maths.uct.ac.za; mbijura@yahoo.com 


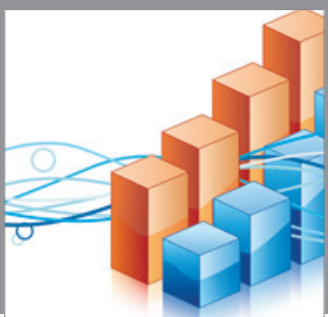

Advances in

Operations Research

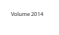

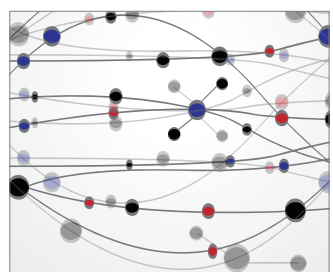

\section{The Scientific} World Journal
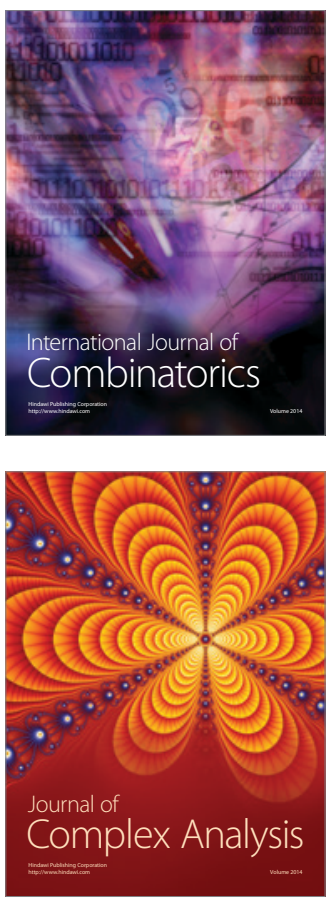

International Journal of

Mathematics and

Mathematical

Sciences
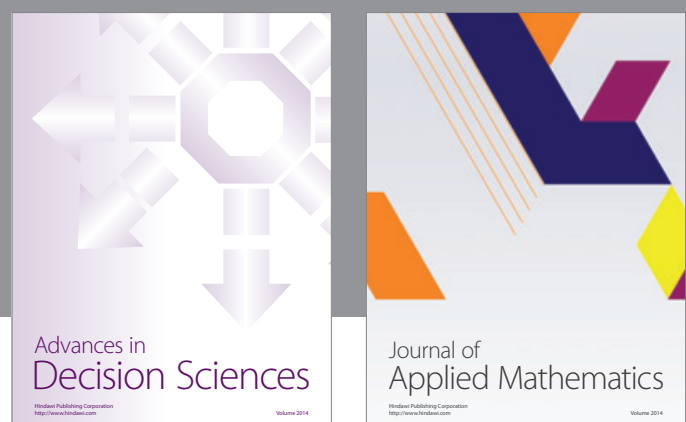

Journal of

Applied Mathematics
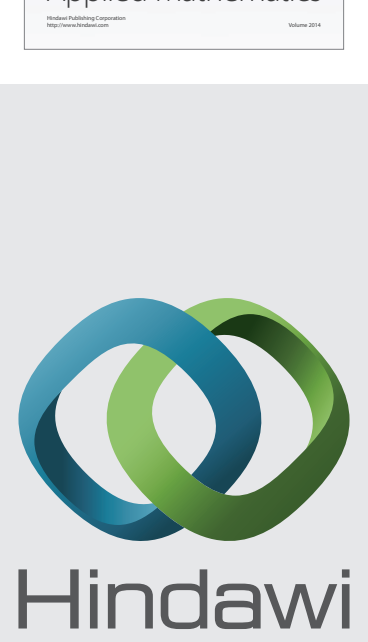

Submit your manuscripts at http://www.hindawi.com
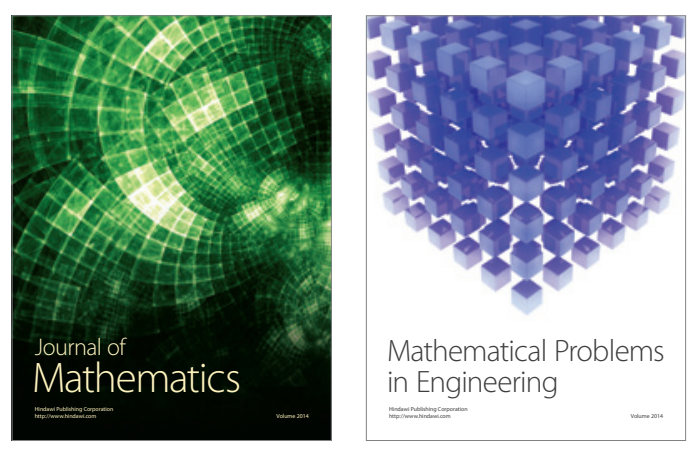

Mathematical Problems in Engineering
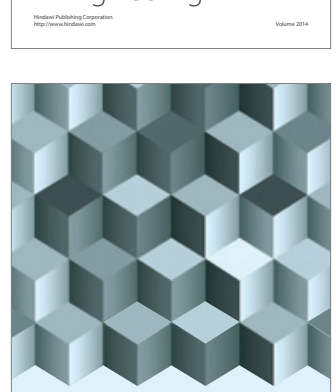

Journal of

Function Spaces
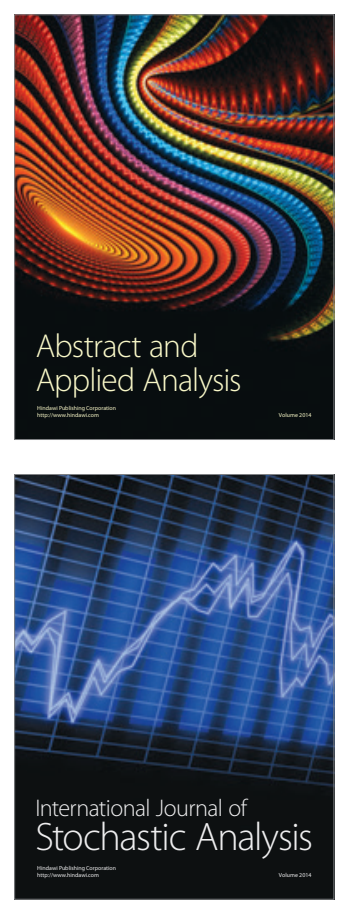

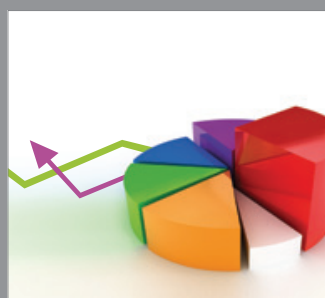

ournal of

Probability and Statistics

Promensencen
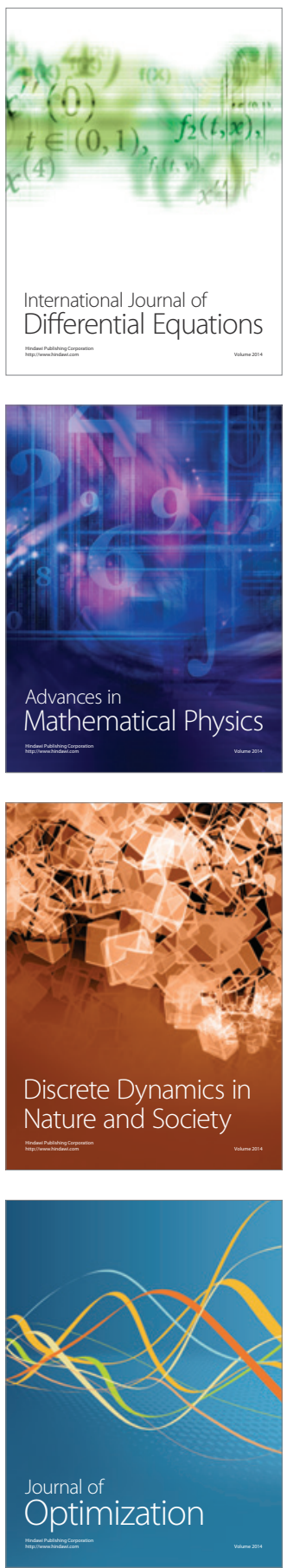